\title{
Correction to: Ambient vibration measurements to support morphometric analysis of a pyroclastic cone
}

\author{
Francesco Panzera ${ }^{1,2}$ (D) - Sebastiano D'Amico ${ }^{3}$ - Emanuele Colica ${ }^{3} \cdot$ Marco Viccaro $^{2,4}$
}

Published online: 30 December 2019

(C) International Association of Volcanology \& Chemistry of the Earth's Interior 2019

Correction to: Bulletin of Volcanology (2019) 81: 74

https://doi.org/10.1007/s00445-019-1338-1

During the correction phase, the publisher inadvertently changed the order of figures 4 and 5 . Therefore, in the article the figure captions are correct, but the figures themselves have been switched. The correct figure order and captions are:

The online version of the original article can be found at https://doi.org/ 10.1007/s00445-019-1338-1

Francesco Panzera

francesco.panzera@sed.ethz.ch

1 Swiss Seismological Service, ETH, Zurich, Switzerland

2 Dipartimento di Scienze Biologiche Geologiche e Ambientali, Università di Catania, Catania, Italy

3 Department of Geosciences, University of Malta, Msida, Malta

4 Istituto Nazionale di Geofisica e Vulcanologia-Sezione di Catania, Osservatorio Etneo, Catania, Italy 

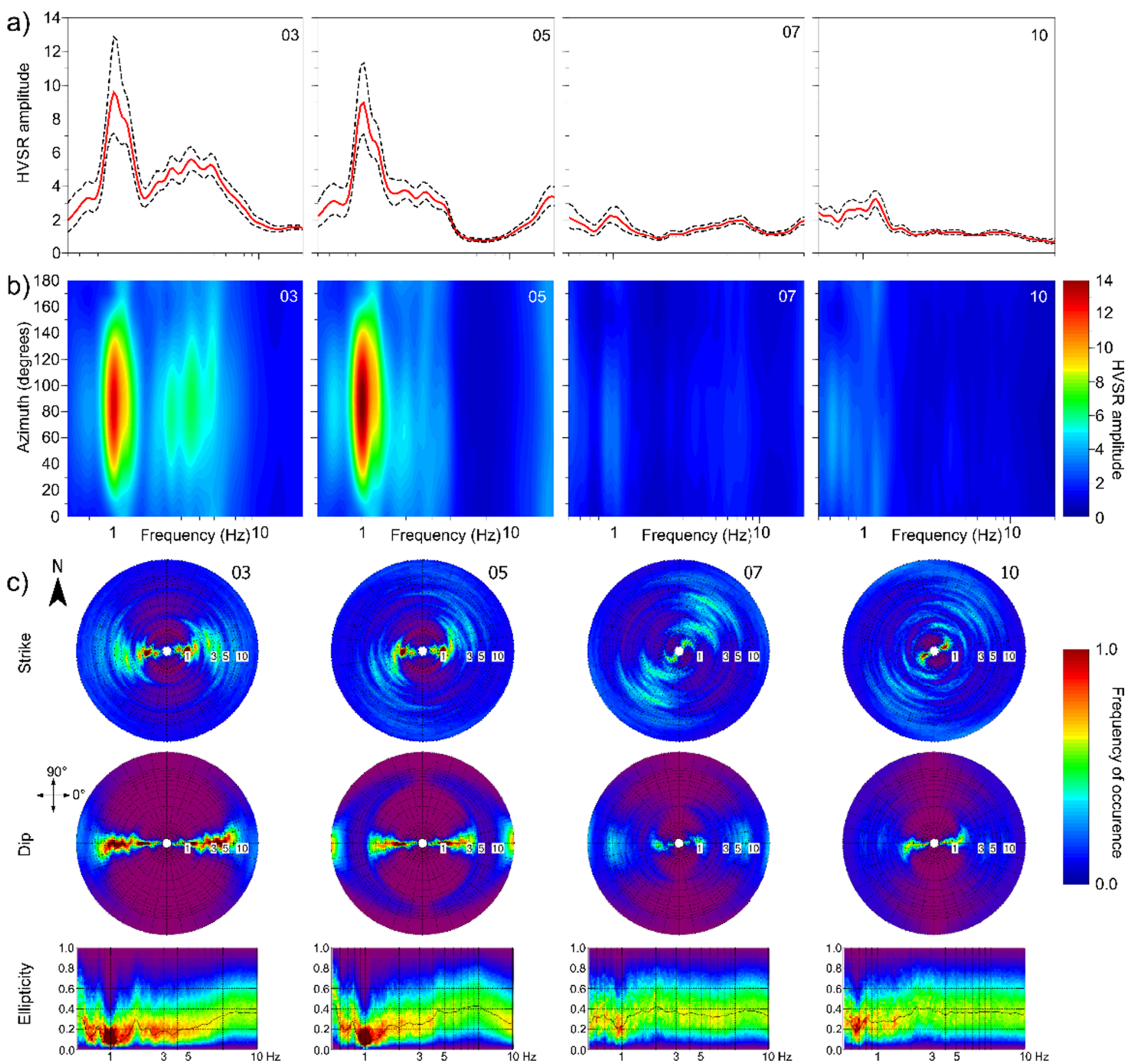

Fig. 4 - a Examples of squared average HVSRs obtained at the investigated sites (red lines mean and dashed black lines standard deviations). HVSRs 03 and 05 were obtained through measurements inside the cone, whereas 07 and 10 were outside (see location in Fig. 1). b Corresponding contour plots of the rotated spectral ratios, plotted
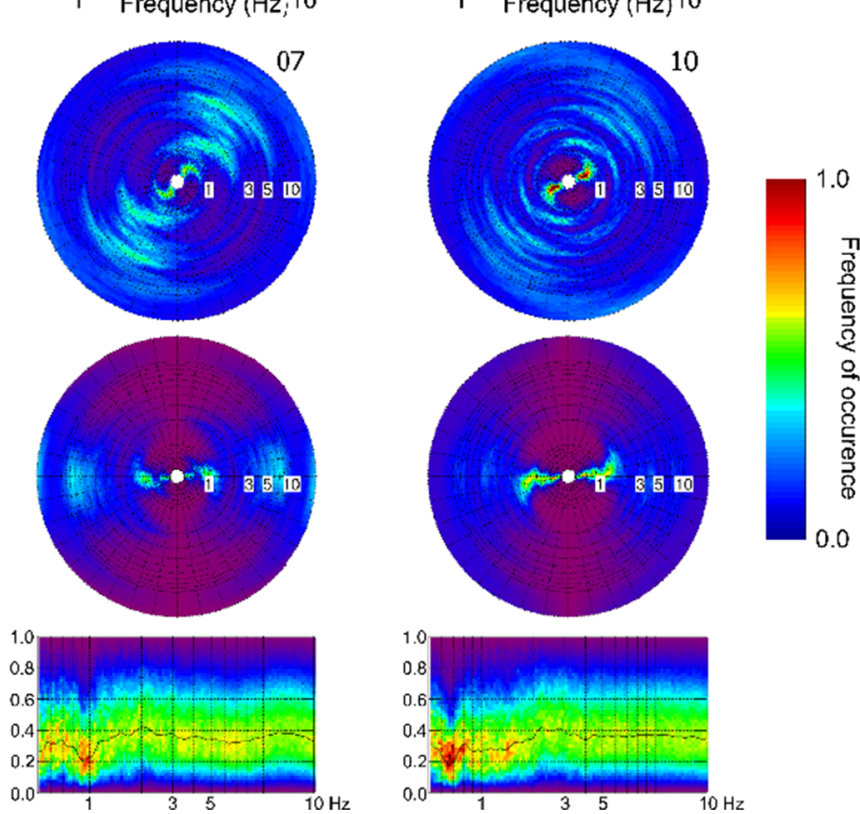

as a function of frequency ( $x$-axis), direction of motion ( $y$-axis), and HVSR amplitudes (scale of colors). c TF analysis results for the selected site in terms of polarization strike (top) and dip (middle) polar plots, along with ellipticity (bottom). 

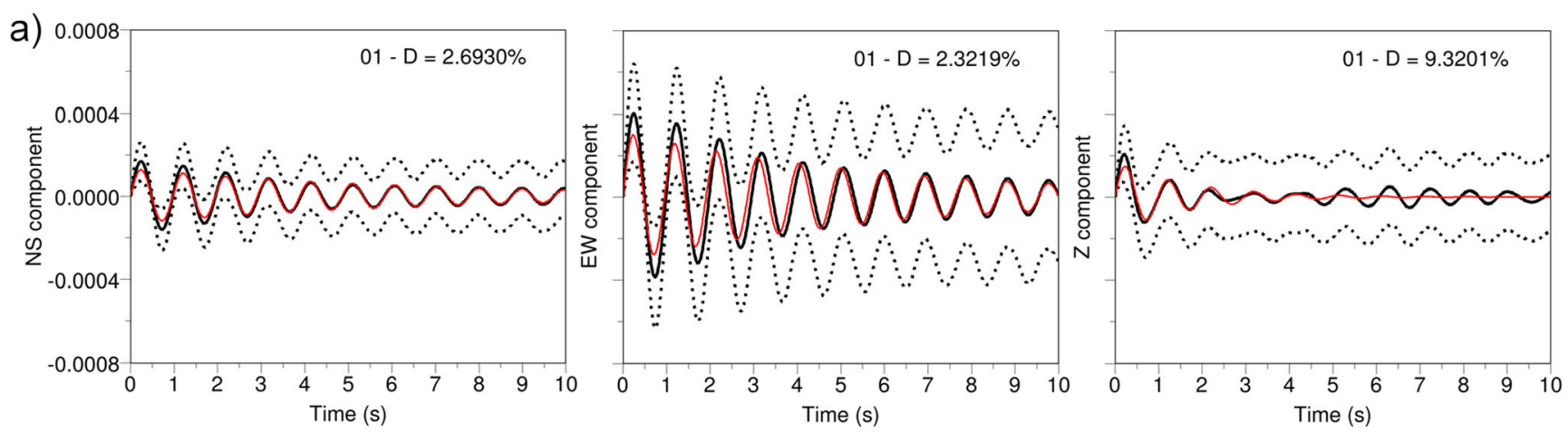

b)
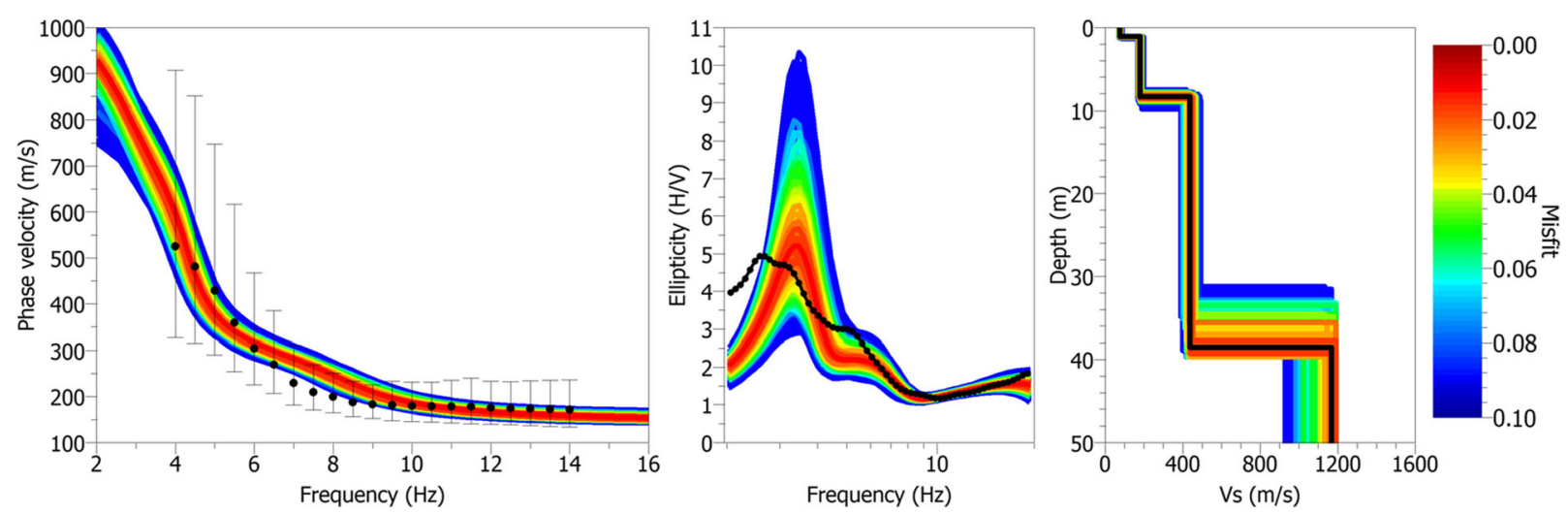

Fig. 5 - a Example of damping computation for the three component ambient vibration measurements performed at the recording site 01 . The continuous black line corresponds to the mean of the RDM, the dashed lines correspond to the standard deviations, and the solid red line is the fitted exponentially decreasing function. b From left to right: inversion results in terms of theoretical dispersion curves, with over-

plotted experimental dispersion curve power spectra maxima (black dots) and their related upper and lower bound limits (vertical black lines); theoretical ellipticity curve, with over-plotted HVSR number 05 (black dots); inversion process results in terms of theoretical shear wave profile, black line denotes the best $V_{\mathrm{S}}$ model. 